$41(1) \mid 2012$

Varia

\title{
La tradición Muitzentza y el Periodo de integración (700-1500 d. C.) en la alta cuenca del río Pastaza, Amazonía ecuatoriana
}

La tradition Muitzentza et la Période d'intégration (7000-1500 ap. J.-C.) dans le haut bassin du Pastaza, Amazonie équatorienne

The Muitzentza Tradition and the Integration Period (700-1500 AD) in the upper Pastaza, Ecuadorian Amazonia

Geoffroy de Saulieu y Carlos Duche Hidalgo

\section{OpenEdition}

Journals

Edición electrónica

URL: http://journals.openedition.org/bifea/1004

DOI: $10.4000 /$ bifea. 1004

ISSN: 2076-5827

Editor

Institut Français d'Études Andines

Edición impresa

Fecha de publicación: 1 enero 2012

Paginación: 35-55

ISSN: 0303-7495

Referencia electrónica

Geoffroy de Saulieu y Carlos Duche Hidalgo, «La tradición Muitzentza y el Periodo de integración (700-1500 d. C.) en la alta cuenca del río Pastaza, Amazonía ecuatoriana », Bulletin de l'Institut français d'études andines [En línea], 41 (1) | 2012, Publicado el 01 agosto 2012, consultado el 06 noviembre 2020. URL : http://journals.openedition.org/bifea/1004 ; DOI : https://doi.org/10.4000/bifea.1004

\section{cc) (†) $\odot$}

Les contenus du Bulletin de l'Institut français d'études andines sont mis à disposition selon les termes de la licence Creative Commons Attribution - Pas d'Utilisation Commerciale - Pas de Modification 4.0 International. 


\title{
La tradición Muitzentza y el Periodo de integración (700-1500 d. C.) en la alta cuenca del río Pastaza, Amazonía ecuatoriana
}

\author{
Geoffroy de Saulieu* \\ Carlos Duche Hidalgo*
}

\begin{abstract}
Resumen
La síntesis de los datos provenientes de diversas colecciones de cerámicas precolombinas, entre las cuales domina aquella del Museo etnoarqueológico de Puyo y Pastaza y de una investigación de campo en la provincia de Pastaza, permite definir la tradición Muitzentza. Se trata de un material poco diversificado que se caracteriza por ollas y tinajas con cuellos, así como también por cuencos que llevan principalmente decoraciones pintadas en blanco sobre rojo, o en blanco, o rojo, sobre fondo natural. Una datación AMS indica un periodo alrededor de los siglos XI-XII d. C., lo que lleva a pensar que se trataría del material de antiguas poblaciones pertenecientes al grupo etnolingüístico zaparoano.
\end{abstract}

Palabras clave: Período de Integración, Amazonía, Záparo, Pastaza, Horizonte corrugado, Horizonte polícromo

\section{La tradition Muitzentza et la Période d'intégration (7000-1500 ap. J.-C.) dans le haut bassin du Pastaza, Amazonie équatorienne}

\section{Résumé}

La synthèse des données provenant de diverses collections de céramiques précolombiennes, parmi lesquelles domine celle du Musée ethnoarchéologique de Puyo et du Pastaza, et d'un travail de terrain dans la province de Pastaza, permet de décrire la tradition Muitzentza. Il s'agit d'un matériel peu

\footnotetext{
* Arqueólogo IRD, UMR 208 «Patrimoines Locaux», IRD-MNHN- Email: geoffroy.desaulieu@ird.fr

** Antropólogo, Director del museo etno-arqueológico de Puyo y de Pastaza, Dirección de educación hispana de Pastaza. Email: carduche@yahoo.com.mx
} 
diversifié, caractérisé par des jarres à col et des bols, portant principalement une décoration peinte en blanc sur fond rouge ou bien en rouge ou en blanc sur fond naturel. Une datation AMS indique une période autour des XI-XIIème siècles ap. J.-C., et permet de penser qu'il s'agit d'un matériel ayant appartenu à d'anciennes populations faisant partie du groupe ethnolinguistique zaparoan.

Mots clés : Période d'Intégration, Amazonie, Záparo, Pastaza, Horizon Corrugado, Horizon Polychrome

\title{
The Muitzentza Tradition and the Integration Period (700-1500 AD) in the upper Pastaza, Ecuadorian Amazonia
}

\begin{abstract}
New fieldwork in the Pastaza region coupled with the study of museum collections, allow us to describe the Muitzentza tradition. The ceramic materials of this tradition are dominated by bowls and necked jars and tinajas decorated in red and white painting. The decoration consists mainly of white painting on red, or red or white on an unslipped surface. An AMS date indicates an age around the XI-XII centuries A.D. It is likely that Zaparoan speakers, historic occupants of the region, are derived from a Muitzentza background.
\end{abstract}

Key words: Integration period, Amazonia, Zaparo, Pastaza, Corrugated Horizon, Polychrome Horizon, Muitzentza tradition

\section{INTRODUCCIÓN}

El río Pastaza es un afluente de la ribera izquierda del Marañón, cuya cabecera se ubica en la cordillera oriental de los Andes del centro del Ecuador, al pie del volcán Tungurahua. La zona de la alta cuenca observada se halla localizada entre el piedemonte y la frontera con el Perú, en medio de la selva amazónica. Por su situación geográfica, el río Pastaza presenta un interés arqueológico particular pues forma parte de los pocos valles ecuatorianos que se encuentran al bajar hacia la llanura amazónica y crean así un vínculo entre las tierras altas de los Andes y las tierras bajas calientes y húmedas. Por otro lado, el río acarrea sedimentos volcánicos que se depositan a lo largo de su curso amazónico. En comparación con el valle del Napo, ubicado más al norte, este eje de circulación ha permanecido probablemente como un eje secundario durante los periodos más recientes, puesto que su navegación se vuelve realmente posible solo $100 \mathrm{~km}$ aguas más abajo.

Los estudios que hemos emprendido en la región entre 2005 y 2009, conciernen un sector en donde las investigaciones arqueológicas anteriores han sido escasas: la comarca de Muitzentza, ubicada a $300 \mathrm{msnm}$. Si bien esta zona está poblada por los achuar, en otros sectores de la provincia de Pastaza existen moradores shuar, pertenecientes también al grupo etnolingüístico amerindio jíbaro. Se encuentran igualmente los kichwa canelos, población de lengua kichwa, cuya etnogénesis es compleja y probablemente ligada a la mezcla entre grupos locales más antiguos y poblaciones desplazadas. En Puyo la población mestiza y de ascendencia 
claramente andina es dominante. Hemos buscado identificar las culturas arqueológicas anteriores a las que identificamos gracias a la etnohistoria (siglos $\mathrm{XVI-XX),} \mathrm{a} \mathrm{fin} \mathrm{de} \mathrm{ver} \mathrm{si} \mathrm{los} \mathrm{datos} \mathrm{arqueológicos} \mathrm{y} \mathrm{etnohistóricos} \mathrm{estaban} \mathrm{conectados.}$

El Período de Integración (700-1500 d. C.) en la Amazonía ecuatoriana, ya sea en Zamora-Chinchipe, Morona Santiago, Pastaza o Napo, empieza por una ruptura cultural: las tradiciones cerámicas y los horizontes estilísticos o culturales (tal como el «flash horizon» de los «Asymmetric Spouts of the Upper Amazon» [DeBoer, 2003: 319-323]) no se inscriben más en un conjunto material continuo con las tierras altas y la costa del Pacífico como era el caso para los periodos anteriores. Al contrario, se ve el asentamiento de grandes horizontes estilísticos foráneos: el Horizonte corrugado por el sur, probablemente traído por el grupo etnolingüístico jíbaro (Guffroy, 2006; Rostain, 1999; 2006; Saulieu, 2006) y el Horizonte polícromo al norte, cuyo conductor parece ser en parte de origen Tupí (Lathrap, 1970).

Este artículo presenta algunos datos arqueológicos nuevos, obtenidos por Carlos Duche Hidalgo y Geoffroy de Saulieu, y los confronta con aquellos, aunque escasos, conocidos en la ribera izquierda del Marañón. Después de situar la región a través de sus características geográficas y etnohistóricas, describe igualmente algunos materiales arqueológicos, para luego abordar las relaciones culturales externas de las cuales este material parece ser testimonio. Por último trata de las posibilidades interpretativas de los datos materiales en términos etnohistóricos.

\section{MARCO GEOGRÁFICO}

El Pastaza nace a 1900 msnm, de la unión de los ríos Patate y Chambo, al pie del volcán Tungurahua (provincia de Tungurahua). El río sigue un valle muy encañonado entre las vertientes de los macizos de los Llanganates al norte y la continuación de la Cordillera Oriental al sur. Su curso está marcado entonces por diversas caídas espectaculares. A partir de $1600 \mathrm{~m}$ de altura, la vegetación se vuelve más tropical: es la selva de nubes, una formación muy densa con árboles de tamaño medio, donde se distinguen los helechos arborescentes y las orquídeas. El encañonado desemboca de repente en el piedemonte a $1100 \mathrm{msnm}$. Mesetas y colinas reemplazan las vertientes empinadas de los Andes y la vegetación se presenta cada vez más tropical: al bajar de altura, las temperaturas se elevan, los árboles son más altos, y las palmas más numerosas. A este nivel, el curso del río es pedregoso y poco profundo, pero sometido a crecidas muy violentas. La tectónica da una configuración particular al curso del Pastaza: después de tener una dirección NO-SE y afluentes en ambas riberas a lo largo de los 30 primeros km desde el piedemonte (ríos Puyo, Palora, Chiguaza), no existen más afluentes en la ribera sur, hasta llegar a la frontera peruana, situada a unos $145 \mathrm{~km}$ aguas abajo. La ribera norte, al contrario, recibe los afluentes siguientes: ríos Copataza, Capahuari y Bobonaza, todos navegables, lo que los distingue del Pastaza mismo en su curso alto. Es evidente que los precolombinos sobrepasaron las desventajas de esta configuración geográfica, pero al mismo tiempo hay que subrayar que esta situación favorece un acceso por la ribera norte desfavoreciendo el acceso por la ribera sur. El Pastaza es navegable en canoa solo aguas abajo de 
Sharamentza. Aguas arriba, la corriente y los remolinos lo tornan peligroso. Este problema se mitiga gracias al hecho de que el Bobonaza, que desemboca al nivel de la frontera con Perú, es fácilmente navegable desde Canelos, lugar situado no muy lejos de Puyo. Esta fue la ruta tradicional de los misioneros desde el siglo XVIII. El río Copataza aunque muy sinuoso, también es navegable. La selva tropical húmeda es típica de la zona. Los suelos y las aguas son bastante diversos: las zonas de tierra firme tienen a menudo una naturaleza arcillosa con potencialidades agrícolas desiguales. En las llanuras, se depositaron limones volcánicos creando así varzeas fértiles que al igual que toda varzea, son inundables y no drenadas; su uso por lo tanto es difícil. Gracias a la actividad sísmica, antiguas varzeas se han levantadas y se encuentran hoy en día fuera de peligro de las crecidas. Numerosas comunidades indígenas viven actualmente sobre estas mesetas en donde practican una agricultura sin barbecho. Existen tal vez tierras antropogénicas, pero a falta de estudios, no se puede concluir si se tratan de verdaderas terras pretas (Duche Hidalgo \& Saulieu, 2009: 28). Los ríos corresponden a la tipología ya descrita en otros sectores de la Amazonía: hay aguas negras, cargadas de taninos y pobres en oxígeno; aguas blancas que acarrean consigo sedimentos andinos, y aguas claras, sobre todo en el piedemonte, que no contienen ni taninos ni sedimentos, dada la velocidad de su formación sobre las estribaciones orientales de los Andes. Antiguamente la región era muy rica en peces; aguas abajo habían tortugas acuáticas tal como lo indican ciertos topónimos y etnónimos. La especie de tortuga gigante Podocnemys expanda era básica en la vida de numerosos indígenas precolombinos, quienes la cazaban por su carne muy apreciada, su carapacho (charapa) usado como escudo de guerra y sus substancias grasas. Los huevos eran cosechados masivamente para extraer un aceite, y los intestinos de los adultos proveían una manteca muy apreciada. Todo estaba almacenado en ceramios. Parece ser que este almacenamiento era importante y duradero (tal vez un año): de una temporada de desove a otra, es decir en cada verano, cuando los animales migran para buscar bancos de arenas calentados por el sol (Trujillo, 2001). Tal vez este sistema haya permitido compensar la irregularidad de la caza (potencial que demora en reconstituirse) y de la pesca (eficiente solo en periodo de aguas bajas). Se puede recordar que la actividad humana, al favorecer directamente o indirectamente a los vegetales comestibles, tal como las palmas chonta (Bactris gasipaes) y muriti (Mauritia flexuosa), ayudaba los animales de caza.

Al cruzar la frontera peruana, el río Pastaza continúa su curso cada vez más sinuoso a lo largo de más de 250 km hasta el río Marañón. Su orientación general es un poco distinta pues tiene un eje más o menos $\mathrm{N}-\mathrm{S}$.

Los datos arqueológicos presentados por el artículo dan cuenta únicamente de la parte ecuatoriana entre el piedemonte y la frontera.

\section{DATOS ETNOHISTÓRICOS}

Los datos etnohistóricos cuyas fechas corresponden al periodo entre los siglos XVI y XX son bastante escasos. Ciertas obras recientes intentan utilizar fuentes primarias principales. Entre estas constan, el trabajo de Taylor (1986) basado en 
Ios archivos coloniales; Trujillo (2001) quien se remite a archivos, mapas, historia regional, antiguas publicaciones, memoria oral y lingüística para reconstruir los procesos históricos culturales de la cuenca del río Curaray; y Reeve (2002) quien hizo una etnografía actual de los kichwas del Curaray. Sin embargo, hay que destacar el hecho de que no existe ninguna etnografía que trate de los záparos antes de su drástica disminución en el siglo XIX (es por eso que se desconoce su cultura material anterior a su kichwisación). Los textos primarios son muy pobres en cuanto a los datos de la cultura material que nos interesa.

Todos señalan que la región ubicada entre las riberas sur del río Napo, norte del río Pastaza y oeste del río Marañón estaba poblada en su mayoría por grupos humanos pertenecientes al grupo etnolingüístico zaparoano (Taylor, 1986; Reeve, 2002; Trujillo, 2001), cuyo origen queda desconocido. Pensamos que este grupo ocupó también la región del piedemonte, aunque no haya pruebas de ello. Para J. Trujillo (2001: 105-107 y comunicación personal) los grupos humanos que subían periódicamente el encañonado del Pastaza hasta las caídas de Agoyán (cerca de la ciudad actual de Baños de las Aguas Santas, al pie del volcán Tungurahua) a fin de cumplir ceremonias rituales, pertenecían al grupo zaparoano. Las caídas de agua tienen todavía una importancia particular entre las poblaciones indígenas del piedemonte ecuatoriano, ya sean de filiación kichwa canelo o de filiación jíbaro (Karsten, 1935; Harner, 1995 [1972]). Se tiene poca información sobre las antiguas poblaciones zaparoanas. Hoy en día en el Ecuador, se habla poco la lengua Zápara (o Sápara), la misma que va camino a su desaparición en el valle del Curaray, al norte del río Pastaza. La lengua de los andoas, probablemente también de filiación zaparoana (Jorge Gómez Rendón: comunicación personal), se encuentra en igual y hasta peor situación. El antiguo término «záparo» podría explicarse por el uso generalizado del caparazón de la tortuga Podocnemys expansa a manera de escudo 1 por los guerreros. Parece ser que las poblaciones de filiación zaparoanas eran diversas. Los documentos históricos mencionan Gaes en la ribera norte del Pastaza (fig. 1. 1), la cuenca del Capahuari y el Bobonaza, Semigaes en la cuenca del Curaray, Záparos en el Alto Tigre, Andoas en el Huasaga y luego en el Bajo Bobonaza. El término Iquitos, correspondiente a una ciudad ubicada en el río Marañón, en la Amazonía peruana, proviene del nombre de un grupo zaparoano. La desaparición de los grupos ha sido compleja y causada por varios factores como son la caza de esclavos río abajo, las guerras generalizadas, las epidemias de contacto, amplificadas a su vez por las «reducciones» y misiones donde se juntaban diversos pueblos, la evangelización realizada en lengua kichwa, etc. El debilitamiento de los grupos zaparoanos, aunque conocidos por su valor guerrero, durante el periodo colonial y republicano, explica la etnogénesis del grupo actual kichwa canelo. La disminución de las fuerzas demográficas y militar zaparoanas, permitió la entrada de grupos de filiación jíbaro en la ribera norte del río Pastaza (fig. 1. 2).

1 Se debe anotar que el nombre de uno de los sitios de donde provienen los materiales de este artículo, «Charapacocha» significa la laguna de las tortugas. Se trataba probablemente de antiguas zonas de recolección de huevos y de caza de tortugas adultas. 


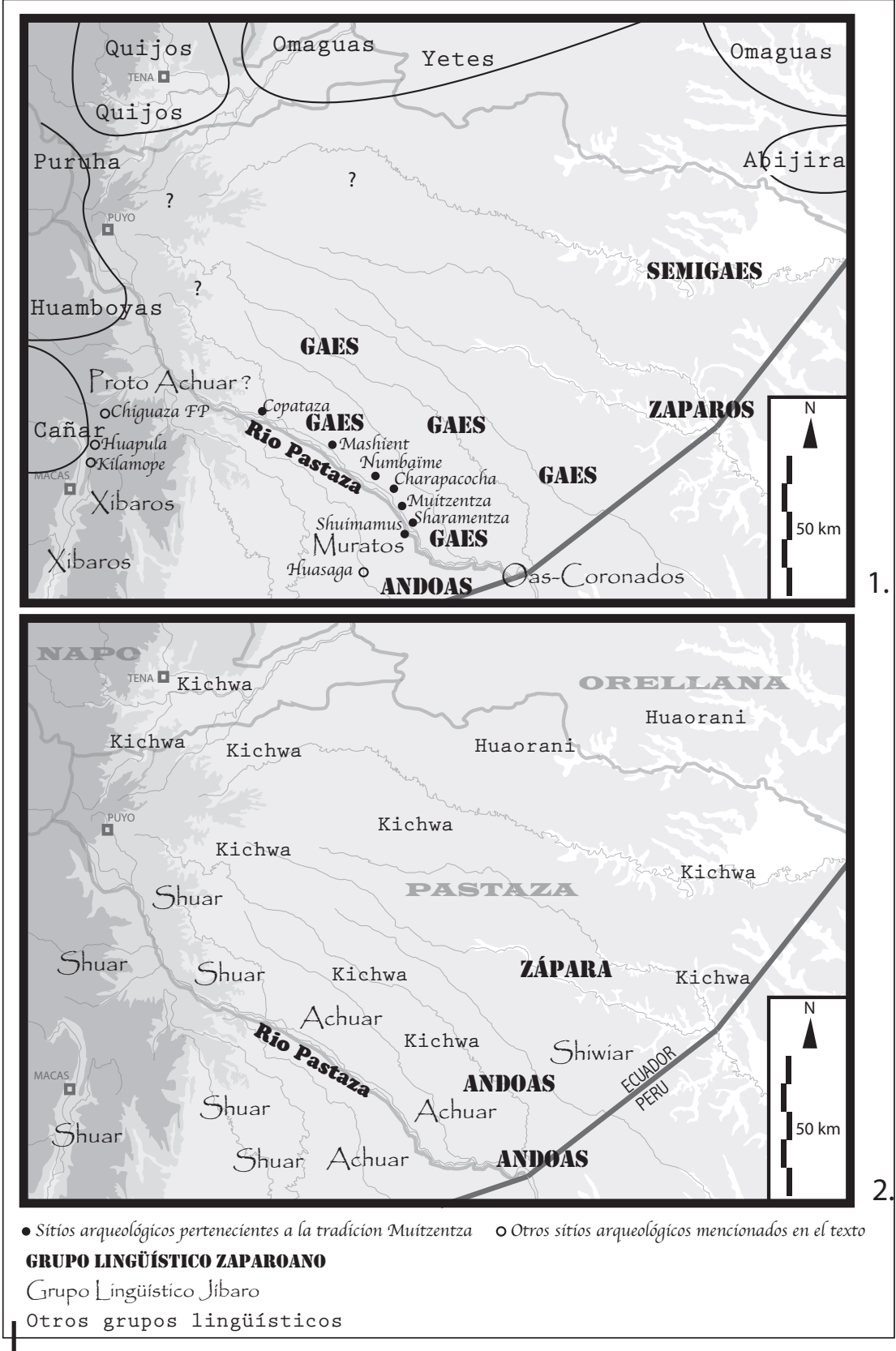

Figura 1 - Mapas de la alta cuenca del río Pastaza

Arriba (mapa 1), la repartición de los grupos etnolingüísticos durante la época colonial (XVIIXVIIIle ss.) según los datos de A. C. Taylor (1986), con los sitios arqueológicos de la tradición Muitzentza. Abajo (mapa 2), repartición de los grupos etnolingüísticos en la época actual: se ve claramente que la desaparición de la mayoría de los grupos zaparoanos favoreció a los grupos jíbaro, así como a los grupos kichwa (dibujos de G. de Saulieu) 
Uno de los problemas concernientes a la etnohistoria de los antiguos grupos zaparoanos reside en el hecho de que se desconoce su cultura material, se ignora también si tenían algunas tradiciones cerámicas distintas de los otros pueblos o entre ellos mismos.

Lo único que se sabe hoy en día de los kichwa canelos, cuya etnogénesis es reciente, es que nacieron construyendo su propio estilo cerámico y que el grupo integró poblaciones zaparoanas.

Tomando en consideración los diversos indicios con los que contamos, parece ser probable que en las comarcas de los actuales poblados de Charapacocha y Muitzentza, los antiguos moradores pertenecientes a los siglos XVII y XIX fueran gaes o grupos desconocidos de filiación lingüística zaparoana.

\section{EL MATERIAL ARQUEOLÓGICO}

\section{1. El corpus}

Los datos arqueológicos disponibles provienen del material cerámico. En este predominan dos modalidades decorativas distintas, aunque comparten características tecnológicas, formas y motivos decorativos comunes. Se ignora aún si las variaciones tienen un sentido cronológico claro. Se requieren todavía más excavaciones, así como un análisis sintético y fino. Podemos identificar ya materiales cuya modalidad decorativa está dominada por la pintura de grandes motivos puestos sobre un fondo de engobe o sobre fondo natural, y otros materiales cuya pintura, aunque mal conservada, fue aplicada entre incisiones para rellenar motivos detallados y de pequeña dimensión. Hay que anotar que numerosos tiestos parecen haber perdido las pinturas que tenían, razón por la cual no vamos a describirlos de manera separada.

La definición de la tradición Muitzentza (topónimo achuar que significa «el río de la tinaja» y que corresponde a la traducción del topónimo español-kichwa «Tinajayacu») se basa en varias colecciones que son, según su orden de importancia:

- la Colección del Museo Etnoarqueológico de Puyo y de Pastaza (18 recipientes cerámicos enteros y 85 tiestos de recolecciones de superficies, provenientes de la comarca de Charapacocha);

- los tiestos de recolecciones de superficie y de excavación de la investigación de campo que se emprendió en diciembre de 2006 en la localidad de Muitzentza, cerca de Charapacocha (100 tiestos diagnósticos y una datación con método AMS);

- diversas colecciones: Casa de la Cultura de Quito (una urna), Misión Dominicana de Puyo (una urna), Fundación Sinchi Sacha en Quito (una urna), una pieza conservada por la propia comunidad de Muitzentza. 


\section{2. Características tecnológicas generales}

La tradición Muitzentza se caracteriza por una pasta con un desgrasante cuyo tamaño es proporcional al espesor de la pared: mayor es el tamaño del objeto, mayor es el espesor del desgrasante, pudiendo llegar hasta la piedrita de $5 \mathrm{~mm}$ de diámetro. Si bien ningún análisis petrográfico se ha realizado, los granos parecen ser de origen local. Generalmente el desgrasante no sobrepasa un $20 \%$ del volumen de la pasta. La técnica de fabricación parece ser bandas de arcilla sobrepuestas (colombin en francés) y posiblemente el modelado para ciertas piezas de tamaño modesto o para los fondos convexos de ciertas urnas de gran tamaño (más de $50 \mathrm{~cm}$ de alto). Aunque no tenemos pruebas, probablemente la técnica utilizada en algunos casos, fue la del martillaje (battage en francés). La superficie externa de los recipientes tiene dos tratamientos principales: engobe clásico (es decir una arcilla distinta foránea y fina puesta sobre la superficie del recipiente antes de su cocción), o lo que llamamos «falso engobe», es decir una nueva humidificación de la superficie externa de la cerámica sobre la que se pasa una piedra pulida muy fina (Duche Hidalgo \& Saulieu, 2009). Tal como lo señala Martineau (2010: 15):

«este procedimiento, más que otros alisamientos, permite comprimir los macroelementos de la pasta al interior de la pared, haciendo aparecer en la superficie las moléculas más finas. El resultado es una pared de excelente calidad gracias a una muy ligera capa superficial de una textura particularmente fina, a menudo mal interpretada como un engobe».

En el Oriente ecuatoriano los mejores resultados de esta técnica se encuentran en la tradición Pastaza-Kamihun (cf. Saulieu, 2006). Se observan también de vez en cuando huellas de regularización de la pared interior de los recipientes si la pasta mantiene una consistencia plástica dura/cuero hasta seca. Esta se realiza por medio de una pepa dura o un canto de río. Las pastas son oxidadas y tienen colores crema, anaranjados y marrón, observándose sin embargo, variaciones de coloración de los tonos claros a oscuros.

\section{3. Las formas cerámicas}

Las formas son pocas. Se encuentran sobre todo ollas y tinajas², ambas con cuello, y cuencos.

El tamaño de las ollas y tinajas varía entre $70 \mathrm{~cm}$ de alto con boca de $65 \mathrm{~cm}$ de diámetro, y $30 \mathrm{~cm}$ de alto para una boca de $25 \mathrm{~cm}$ de diámetro. Es muy probable que existan recipientes más grandes y más pequeños, siendo la forma más corriente la de un cuerpo globular con fondo convexo redondo o convexo ligeramente

2 Para evitar confusiones en la terminología, utilizamos la palabra «olla» para los recipientes de cocina (casi siempre sin decoración pintada) y el término «tinaja» para los recipientes cerrados con cuello y con pintura. No obstante, hay que subrayar que dado el estado fragmentado e incompleto del material, se torna difícil hacer una distinción clara entre los dos términos. 
puntiagudo, cuello rectilíneo divergente o vertical, borde simple o doblado al exterior (fig. 2. 1-4). Existen además otras formas como por ejemplo aquella con cuello convergente y borde evertido no decorado (fig. 2. 6). Otro caso sería el de olla sin cuello no decorado. Varios pedazos de bases planas provienen de ollas de gran tamaño. Las ollas con cuello tienen dos características bastante comunes:

- un canal de 2 a $3 \mathrm{~cm}$ de alto y $1 \mathrm{~cm}$ de profundidad, ubicado en la junción entre el cuello y el cuerpo del recipiente (fig. 2.1 y 3);

- un cambio de inclinación y espesor de la pared en el tercio inferior del cuerpo (fig. 2. 1, 3 y 4), el cual aumenta o disminuye el volumen total de la vasija y marca sobre todo el inicio de la zona decorada en los dos tercios superiores del cuerpo.

El labio de las ollas de gran tamaño se encuentra a menudo aplanado, como si hubiese sido hecho para recibir una tapa de corteza o de hojas. También se ven labios de perfil convexo y adelgazado, o biselado hacia el interior o el exterior,

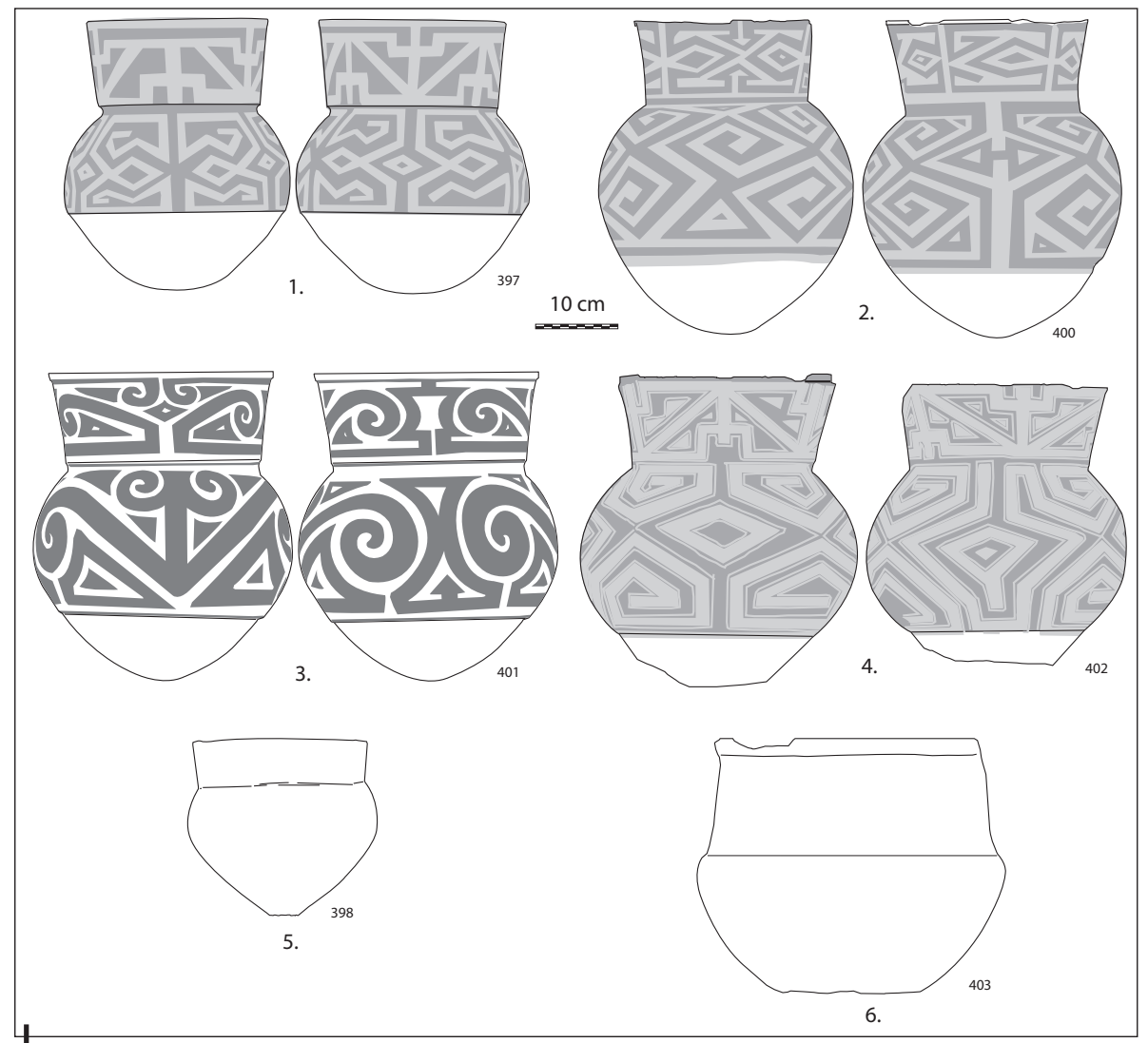

Figura 2 - Ollas con cuello de la tradición Muitzentza

Las 1, 2, 3, 4 y 6 fueron probablemente usadas como urnas funerarias. Colección del Museo etnoarqueológico de Puyo y del Pastaza (dibujos de G. de Saulieu) 
o modificados por modalidades decorativas (ver más abajo). Parece ser que los recipientes de cocina son pocos o no decorados (por ejemplo los cuellos no tienen decorado con base en modulaciones de la técnica corrugada como se ve en Zamora Chinchipe). La vajilla utilizada probablemente para almacenar y tomar chicha es en su mayoría decorada.

En lo que corresponde al número total de cuencos enteros, se puede ver una gran variedad de ellos. En cinco cuencos del museo de Puyo, el alto varía entre 12 y $6,7 \mathrm{~cm}$, y la boca entre 18 y 13,5 cm de diámetro, mientras que las bocas de los cuencos provenientes de los sondeos oscilan entre 30 y $20 \mathrm{~cm}$ de diámetro. Se cuentan cuatro (o cinco) casos distintos, uno solo proveniente de los trabajos arqueológicos. Todos tienen un labio con sección convexa y adelgazada:

- cuencos con pared y borde directo (colección del museo y material de sondeo), no se conoce uno solo entero, pudiendo ser la forma original aquella del cuenco con pared que presenta una ruptura en la línea de inclinación para, de esta manera, disminuir el volumen total del recipiente. Su base es convexa (fig. 3. 1);

- un cuenco ligeramente cerrado (el ancho máximo del recipiente sobrepasa $4 \mathrm{~cm}$ el diámetro de la boca), con pared redondeada y una base casi plana (fig. 3. 2);

- dos cuencos con borde marcado y alto (el alto es superior a la tercera parte del alto total), rectilíneo, divergente y separado del resto por un canal; tienen una base plana y una convexa (fig. 3. 3);

- un cuenco con borde rectilíneo y divergente cuyo alto es superior a la tercera parte del alto total (fig. 3. 4).

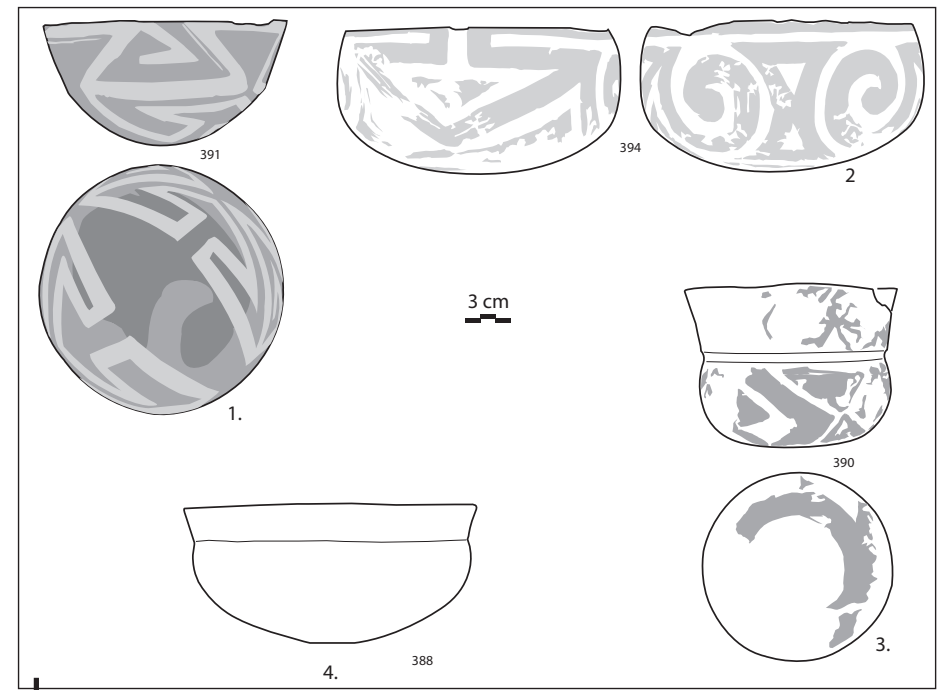

Figura 3 - Las cuatro formas de cuencos enteros conocidos para la tradición Muitzentza

Colección del Museo etnoarqueológico de Puyo y del Pastaza (dibujos de G. de Saulieu) 
Debemos subrayar que no se han encontrado ciertas formas como la plancha cerámica para cocinar la mandioca amarga y que se halla en las tradiciones subactuales y arqueológicas de la Amazonía baja; tampoco hay botellas como en las tradiciones más antiguas de la Alta Amazonía (DeBoer, 2003; Duche Hidalgo \& Saulieu, 2009: 33).

\section{4. Las técnicas decorativas}

La técnica decorativa más conocida registrada en nuestros datos es la pintura blanca en banda de 2 o $3 \mathrm{~cm}$ de ancho, sobre fondo de engobe rojo (fig. 2. 1, 2 y 4 ; fig. 3. 1). Se observan ciertas variaciones: pinturas con bandas iguales en un primer caso, de color o blanco o rojo, aunque sobre fondo natural (fig. 2. 3; fig. 3. 2 y 3). Y en otros casos particulares, bandas blancas sobre fondo rojo, aunque estas tengan de cada lado una línea blanca (fig. 2. 4).

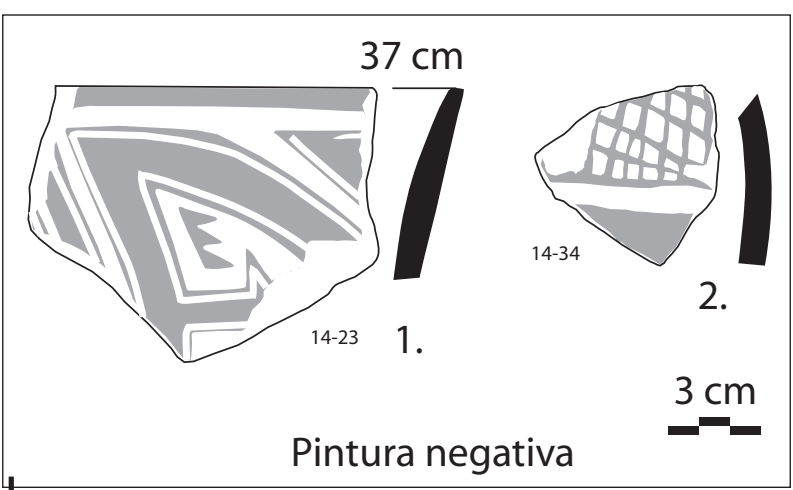

Figura 4 - Dos tiestos de ollas con decoración de pintura negativa

Material proveniente de sondeos realizados en el sector del poblado actual de Muitzentza (dibujos de G. de Saulieu)
La decoración cubre los dos tercios superiores de la olla. Se detiene en la línea de cambio de inclinación y de espesor de la pared cerca del fondo. En lo que tiene que ver con los cuencos, su decoración cubre la casi totalidad de la superficie exterior, mientras que el interior nunca tiene decoración, como se lo puede apreciar en la vajilla kichwa canelo actual.

Se conocen casos de pintura negativa (fig. 4) a pesar de que esta técnica no parezca ser muy corriente.

En la colección del museo de Puyo, se encuentra una modalidad muy importante, la misma que casi está ausente del material de los sondeos: se trata de la pintura roja y/o blanca puesta sobre motivos cuyo contorno es inciso con línea fina y poco profunda (en promedio: 0,5 $\mathrm{mm}$ de profundidad y de ancho, aun si ciertos recipientes de gran tamaño tenían incisiones de hasta $2 \mathrm{~mm}$ de ancho) realizada sobre pasta todavía húmeda (fig. 5). Las incisiones están acompañadas en un caso por impresiones de círculos (diámetro de $0,5 \mathrm{~cm}$ ) probablemente hechas con un carrizo (fig. 5. 1).

Numerosos tiestos sin color presentan las características descritas anteriormente. Es muy probable que hayan perdido su pintura.

Otros llevan decoración corrugada, hecha con bandas de arcilla sobrepuestas (colombin en francés) y que permanecen visibles en la superficie exterior del recipiente. Muy a menudo se trata de una decoración que aparece en los cuellos de las ollas o sobre las partes superiores de los cuencos. En la mayoría de los 


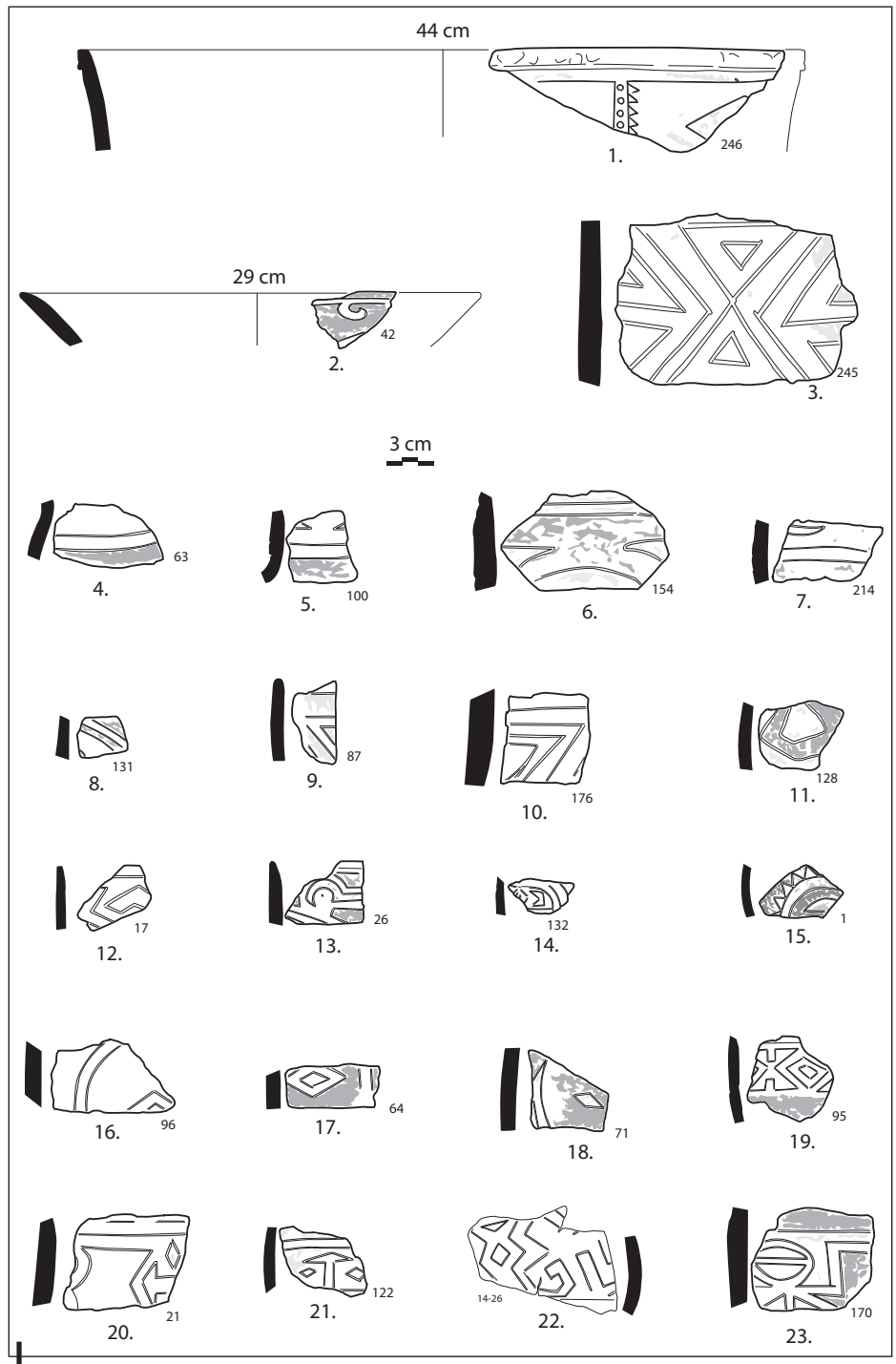

Figura 5 - Tiestos con la modalidad decorativa de pintura entre incisiones

El tiesto 22 proviene de un sondeo realizado en el sector del poblado actual de Muitzentza. Los demás pertenecen a la colección del Museo etnoarqueológico de Puyo y del Pastaza (dibujos de G. de Saulieu)

casos, el corrugado es una etapa de la fabricación, hecho con rollos de arcilla que se unen por presión de los dedos. En varias tradiciones cerámicas de la Alta Amazonía, se aprovecha de esto para crear una decoración donde se juega con presión variable de los dedos, incisiones de uñas, trazos transversales y superficiales con un dedo, incisión y puntuación hechas con un palito, impresión de materias vegetales (cortezas), etc. Aquí, observamos dos casos de tiestos corrugados 
sencillos, uno de corrugado con pintura roja proveniente de una olla, y otro de corrugado fino (colchones de $0,5 \mathrm{~cm}$ de alto) y aplastado proveniente del cuello de una urna de gran dimensión (diámetro de la boca de $45 \mathrm{~cm}$ ). Se diría que la fabricación es local dada la textura de las pastas que no parecen ser muy distintas de las otras cerámicas. La utilización de pintura sobre un corrugado, como en el caso mencionado, es poco frecuente. No es característica de la tradiciones arqueológicas que pertenecen al Horizonte corrugado tal como lo define Guffroy (2006). Creemos estar aquí frente a un caso de adaptación local de una modalidad decorativa más bien foránea, tema que abordaremos más adelante.

Las decoraciones en «apliqué» aparecen solo sobre un tiesto (fig. 6. 4). Este tiene una banda en zigzag con puntuaciones impresas sobre pasta fresca, la misma que se halla

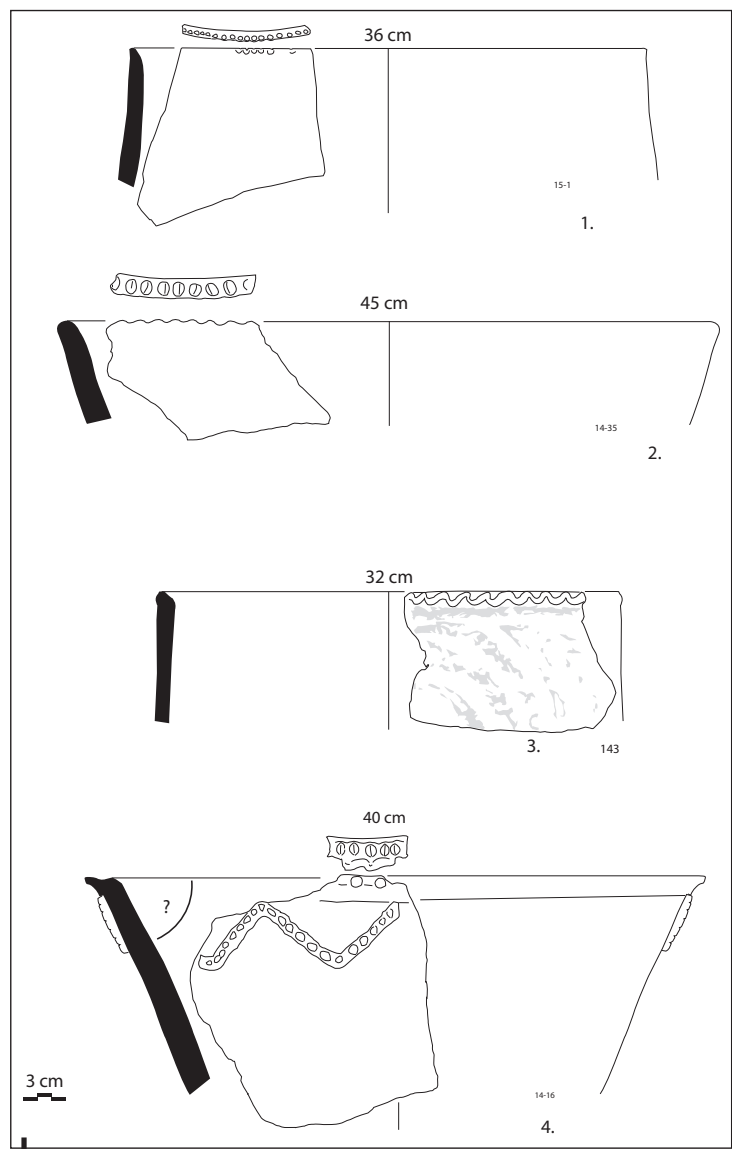

Figura 6 - Fragmentos de cuellos de ollas de gran dimensión, con diversas modalidades decorativas relacionadas con la boca del recipiente

El tiesto 3 pertenece a la colección del Museo etnoarqueológico de Puyo y del Pastaza, mientras los demás provienen de sondeos realizados en el sector del poblado actual de Muitzentza (dibujos de G. de Saulieu) dispuesta debajo del borde doblado hacia el exterior y decorado con impresiones circulares regulares de una olla.

Finalmente, no es extraño ver labios de ollas decoradas: puntuaciones realizadas sobre pasta fresca con un segmento vegetal (fig. 6. 1), ondulaciones obtenidas por medio de impresión regular del dedo (fig. 6. 3) en el borde doblado, impresión de la extremidad del dedo y de la uña en el labio (fig. 6. 2).

\section{5. Los motivos decorativos}

Los motivos decorativos son geométricos. Se pueden observar variaciones en los motivos en relación con la técnica decorativa utilizada.

En lo concerniente a los ceramios pintados, existen dos estilos decorativos que podemos resumir de la siguiente manera:

- un estilo a base de bandas rectas, ángulos, triángulos, rombos y motivos en doble flecha (ejemplos : fig. 2.1 y 2 ; fig. 3. 1);

- un estilo a base de bandas rectas y espirales, rombos, flechas y figuras con dos lados cóncavos (ejemplo: fig. 2. 3; fig. 3. 2).

El primero está vinculado con la técnica de pintura sobre engobe, mientras que 
el segundo se halla en relación con la pintura sobre fondo natural. Existe por lo menos una excepción de pintura sobre engobe que utiliza espirales, lo que muestra que se trata solo de una tendencia.

Los tiestos más fragmentados que llevan decoraciones pintadas entre incisiones presentan líneas rectas (fig. 5. 4, 5, 9, 10), triángulos (fig. 5. 1, 3, 15), rombos (fig. 5. 1, 14, 17, 18, 19, 20, 21, 22), figuras en flechas (fig. 5. 20, 21), discos rayados (fig. 5. 23), figuras en X (fig. 5. 19).

En este último estilo, es cierto que los motivos se asocian de manera diferente al otro estilo pintado sin incisiones. Sin embargo numerosos motivos son iguales en los dos estilos. Por ejemplo, la figura en X aparece también en el cuello de una olla con pintura blanca sobre fondo rojo (fig. 2. 2). La figura en doble flecha (ver la panza de la fig. 2. 2, derecha), no aparece claramente en el estilo de decoración pintada entre incisiones. Probablemente eso se explique por la fragmentación del material (en la fig. 5. 20, podemos ver un pedazo probable de una doble flecha con un rombo).

Gracias a la combinación de los motivos y a los contrastes entre colores, ciertas tinajas grandes, pintadas sobre fondo de engobe, revelan figuras antropomórficas (rostros), ya sea sobre el cuello (fig. 2. 2, izquierda), o sobre la panza (fig. 2. 1, izquierda). Estos rostros no son nunca explícitos y se inscriben dentro de una tradición gráfica fuerte, sin ser iguales tampoco. Por esta razón, los motivos, siempre geométricos, no presentan necesariamente una simetría perfecta. Se vuelve difícil distinguir el inicio y el final de un motivo que se encadena: «el aspecto laberíntico» como lo escribe Anne Christine Taylor (2010: 48) «atrapa la mirada en la trampa» y constituye «un inicio de procesos mentales a fin de engendrar cierta forma de imaginación visual» (traducción nuestra). Vale subrayar que para Taylor, este tipo de decoración es característico de las sociedades amazónicas cuyas ontologías son anímicas (Taylor, 2010: 48).

\section{6. Repartición geográfica, datación y correlaciones cronoestilísticas}

Por lo que conocemos hoy en día, la tradición Muitzentza tiene una repartición geográfica concentrada en un segmento de la alta cuenca del Pastaza: entre Copataza y Shuimamus, es decir en unos 70 km de largo (fig. 1. 1). Con excepción de Shuimamus, todos los sitios se encuentran en la ribera norte, la misma que recibe la casi totalidad de los afluentes. Se ignora aún si las cuencas de los ríos Bobonaza y Capahuari fueron ocupadas por los mismos grupos precolombinos. Lo que parece claro es que los portadores de la tradición Muitzentza tenían una tendencia fluvial, a la vez que una atracción por los caminos. En efecto, es en esta zona que el Pastaza se vuelve navegable río abajo, siendo reemplazado aguas arriba por los caminos.

Unos trabajos de campo en diciembre de 2006, cerca de Muitzentza, permitieron hacer una datación con método AMS, financiada por Stéphen Rostain (CNRS) a 
quien agradecemos nuevamente aquí. El resultado calibrado indica un periodo situado entre 1025 y 1172 d. C., Lyon-4608 (SacA-9666), y proviene de un basural en el fondo de un pequeño valle, al pie de una colina, sellado por una capa totalmente estéril de $40 \mathrm{~cm}$ de espesor. Es verdad que el contexto no es el óptimo, dado que no se trata de un evento (tipo fogón o depósito intencional) que es la junta de pedazos de carbonos recogidos entre $43 \mathrm{~cm}$ y $85 \mathrm{~cm}$ de profundidad (fig. 7). Aún así, lo consideramos relativamente bueno ya que el material es muy homogéneo, y además la capa estéril que lo sella parece haber caído muy rápidamente (a manera de deslave) de la vertiente de la colina.

De forma global, el resultado parece correcto al compararlo con lo que conocemos a nivel regional. En efecto, la presencia de tiestos decorados con la modalidad corrugada concuerda con el Horizonte corrugado (Guffroy, 2006: 350-353), característico del periodo de Integración en la provincias de Morona Santiago, Zamora Chinchipe y Loja. Tal como ha sido precisado anteriormente, estos tiestos, si bien no son numerosos en el material de excavación, parecen ser de fabricación local.

La cronología interna de la tradición Muitzentza resulta más problemática. Las variaciones entre materiales con decoración pintada sobre fondo de engobe o fondo natural, y materiales con decoración pintada entre incisiones llama a varios comentarios. No se puede confundir el estilo con incisiones con otras tradiciones más antiguas. En efecto,

- no tiene nada que ver con el Pastaza-Kamihun, dado que este último grupo se caracteriza por incisiones finas $(1 \mathrm{~mm}$ ) y profundas (hasta $1,5 \mathrm{~mm}$ ) en pasta generalmente fina y homogénea, con zonas excisas y achuradas, una superficie pulida y sin pintura (cf. DeBoer et al., 1977; Saulieu, 2006, Duche \& Saulieu, 2009: 52-57 y 77-89);

- es distinto de la tradición Tinajayacu fechada entre los siglos II y VI a. D. (cf. Duche \& Saulieu, 2009: 30-39) que presenta pastas distintas, paredes más finas, formas diferentes (por ejemplo carenas que no aparecen aquí), motivos decorativos donde dominan escalones, laberintos muy finos, grecas, zigzag, líneas muescadas, y nunca rombos, flechas, triángulos, círculos rayados ni figuras en X.

- es semejante al estilo de Muitzentza sin incisión por las pastas, algunas formas (cuello de tinaja, borde de cuenco), motivos decorativos con rombos, figuras en $\mathrm{X}$, flechas, triángulos. Conocemos únicamente piezas enteras con el estilo pintado sin incisión; el material que lleva pintura entre incisiones se halla demasiado roto y desgastado.

Todo eso nos conduce a pensar que el estilo que asocia pintura e incisiones tiene un parentesco estrecho con el otro estilo, pudiendo incluso ser más antiguo. Esto explicaría el por qué de su casi inexistencia en los dos sondeos realizados en el basural de Muitzentza (único ejemplar en la fig. 5. 22). 


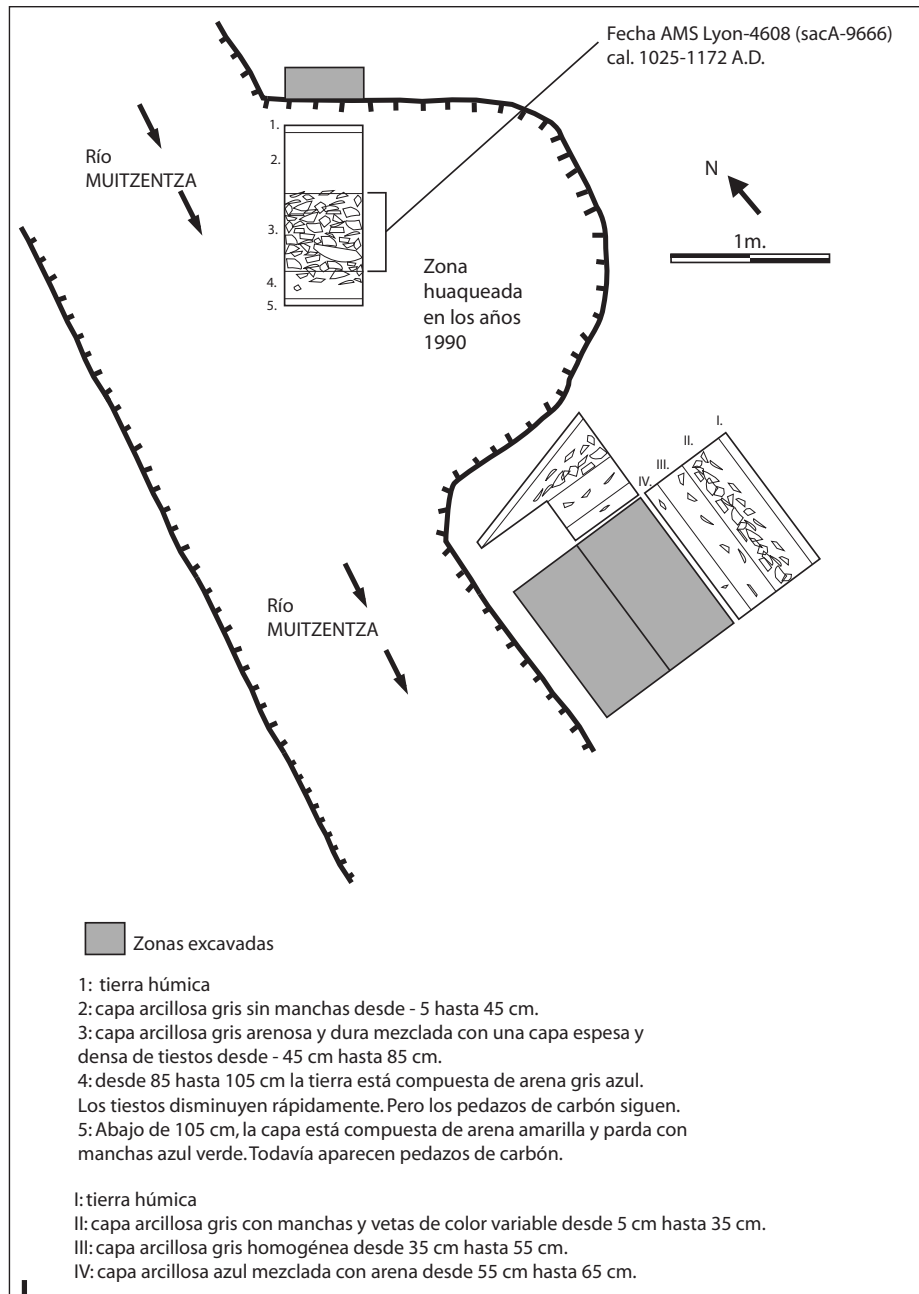

Figura 7 - Situación de los sondeos (diciembre de 2006) en la orilla del río Muitzentza, sector de las cabañas de Suritiak

(dibujos de G. de Saulieu)

\section{COMPARACIONES}

\section{1. Prácticas funerarias}

Los achuar de las comarcas de Charapacocha y Muitzentza afirman que la grandes ollas pintadas, como las del Museo etnoarqueológico de Puyo eran urnas funerarias, dado que en el interior de varias de ellas, han encontrado huesos humanos. No logramos verificar esas observaciones en el campo y no conocemos detalles. Probablemente servían como entierro secundario, lo que no impidió que 
la urna sirviera anteriormente de olla para la fermentación de gran cantidad de chicha destinada a las fiestas colectivas. Debemos subrayar que, del punto de vista simbólico, la fermentación que hace pasar una mezcla de yuca masticada y agua, al estado de bebida alcohólica, podría referirse al paso de la vida a la muerte, o de la muerte al más allá. Este tipo de práctica funeraria que utiliza ollas es bastante común tanto en los datos etnológicos subactuales, como en los arqueológicos de las regiones pertenecientes a los horizontes corrugado ubicados al sur del Pastaza, o polícromo en el norte, a lo largo del río Napo. Se torna imposible, pues, tomar el criterio del uso de las ollas funerarias a fin de establecer comparaciones.

\section{2. Horizonte corrugado: influencias meridionales directas}

Las influencias directas provenientes del Horizonte corrugado tal como lo define Guffroy (2006) son visibles. Lo ilustran los tiestos que llevan esta modalidad decorativa. Algunos de ellos podrían ser una reinterpretación local. En efecto, el corrugado juega con una decoración en relieve hecha con bandas de arcilla que se dejan visibles al exterior del recipiente. En las provincias de Morona Santiago (Rostain, 1999; Saulieu, 2006, cf. fig. 1. 1), de Zamora Chinchipe y de Loja (Guffroy, 2006), esas decoraciones son moduladas con apliques, huellas de dedo, huellas de uñas, incisiones, impresiones de corteza. Junto a esta vajilla corrugada, encontramos una vajilla de tamaño más modesto, decorada con engobe y pinturas, solamente con incisiones o sin decoración alguna. Sin embargo, en todos los casos, la pintura sobre corrugado fue una modalidad no muy común.

En Muitzentza se observa al menos un tiesto corrugado (cuello de olla) y pintado de rojo. Esta particularidad recuerda otro caso en la colección Huasaga de Porras (Saulieu, 2006: 295), un sitio ubicado a unos kilómetros de Muitzentza. Se trataría tal vez, de una mezcla en zona fronteriza, aunque es verdad que la muestra resulta insuficiente para adelantar conclusiones.

Otro caso es el de un pedazo de cuello de olla de gran tamaño que tiene debajo del labio una banda en aplique de arcilla en zigzag y puntuada de impresiones circulares. Este tipo de decoración se encuentra en materiales arqueológicos del mismo periodo, provenientes de Loja, de Zamora Chinchipe en el Ecuador, y en las regiones peruanas de Amazonas (cuenca del Abiseo, un afluente del Huallaga, también en el sector de Kuelap en Chachapoyas, cf. Guffroy, 2006; Duche Hidalgo \& Saulieu, 2009). Los «jeberos», una población subactual ubicada en la unión del Huallaga y del Marañón entregaba hace poco, material cerámico comparable (Tessmann, 1999 [1930]: 238, fig. 9).

\section{3. El Horizonte polícromo: influencias indirectas}

Las influencias provenientes del Horizonte polícromo, contemporáneo del Horizonte corrugado y de la tradición Muitzentza son difíciles de evidenciar ya que nada lo prueba, ni las formas, ni las decoraciones. En efecto, el Polícromo 
de la alta Amazonía que se ilustra en el Ecuador con la fase Napo (Evans \& Meggers, 1968), en el Ucayali peruano con la tradición Caimito (Lathrap, 1970), en Colombia con la fase Zebú (Bolian, 1976), se caracteriza por una gran variedad de formas de recipientes, con bordes a menudo almenado, bocas a veces cuadrangulares, decoraciones muy complejas cuya sutilidad juega sobre distintas técnicas decorativas. Además las decoraciones se encuentran a menudo en el interior de los recipientes abiertos, tales como cuencos o platos, lo que no ocurre con el material de Muitzentza.

A pesar de ello, nos preguntamos si la elaboración de la tradición Muitzentza no se hizo, en parte al menos, en contra de la tradición polícroma. Hay que recordar que las tradiciones polícromas en la última época precolombina jugaron tal vez un papel de candado en alta Amazonías. El complejo Muitzentza, al utilizar pinturas y engobes, hace una demostración de maestría técnica y artística. Ilustra, tanto como las tradiciones polícromas, «el estetismo del gesto» artesanal (Taylor, 2010: 48, traducción nuestra), y su aspecto laberíntico en Muitzentza como en la tradiciones polícromas «pierde a la mirada» (Taylor, 2010: 48, traducción nuestra). La diferencia entre las dos tradiciones se ubica en la austeridad de Muitzentza: al contrario de la tradición polícroma, las alfareras (o alfareros) de la alta cuenca del Pastaza rechazaron las decoraciones exuberantes así como el representar el cuerpo humano o ciertos segmentos de este. En el Napo por ejemplo, no es raro ver decoraciones cerámicas y formas modeladas zoomorfas o antropomorfas (a menudo se trata de urnas funerarias en forma de personaje sentado sobre un banco). Esta costumbre parece estar ausente en la alta cuenca del Pastaza. Ciertas decoraciones pueden tener una clara semejanza antropomorfa pero únicamente de manera metafórica.

\section{4. Insuficiencia de los datos aguas abajo}

Aguas abajo de la zona estudiada, las informaciones son escasas y dispersas. DeBoer menciona el sitio del lago Anático (DeBoer et al., 1977), situado en el bajo curso del Pastaza, en la ribera de una zona lacustre, del cual proviene un recipiente decorado con motivos geométricos incisos. Este recipiente aislado parece muy distinto del material que tenemos, tanto por su forma como por sus motivos decorativos.

Existen informaciones esporádicas provienentes de diversas zonas peruanas vecinas, en los afluentes de la ribera izquierda del Marañón: río Corrientes (Fung Pineda, 1981; Ravines, 1981), río Morona (Ravines, 1989; Rivas Panduro et al., 2008), río Chambira (Morales Chocano, 1991; Myers \& Dean, 1999). Igual que en el Ecuador,

3 En efecto, la fase Napo controla el valle del Napo al norte, acceso directo a la sierra ecuatoriana, rica en oro y tortugas; Caimito, al sur, controla uno de los accesos a las altas tierras andinas peruanas, en donde debemos resaltar la presencia de las minas de sal del Ene y Perené; y la tradición Zebú, ubicada en el río Marañón, después de la junta del sistema fluvial situado entre el Napo y el Ucayali, que desemboca en la Amazonía media. 
los datos están a menudo desprovistos de los contextos o aparecen aislados. Pese a estos inconvenientes, tenemos la impresión de que mientras más nos aproximamos al Marañón, mayor es la diversificación de los materiales arqueológicos.

Vale mencionarse aquí la «fase Tigrillo», descrita por Daniel Morales Chocano (1991), la misma que se intercala entre la «fase Siamba», con influencias barrancoides, y la «fase Urarina» que se puede atribuir al presente etnográfico. El material está compuesto por una cerámica cuya decoración se divide entre el corrugado, el «bicromo inciso» (pintura negra o roja entre incisiones) y el «polícromo sin incisión» (negro y rojo). En esta última, los motivos descritos en el artículo, hechos con bandas pintadas, conducen a pensar en el estilo de Muitzentza elaborado con pintura sobre fondo engobado o fondo natural. Se observan por otro lado, bandas que forman motivos triangulares.

Un hallazgo reciente atestigua una relación directa entre las poblaciones antiguas de los dos lados de la frontera actual. Se trata de un objeto de arcilla, proveniente de la localidad de Huagramona (Rivas Panduro et al., 2008), ubicada a orillas del río Pastaza peruano. Tiene una forma rectangular y lleva motivos pintados en rojo y blanco entre incisiones. No se indican al pie de la fotografía del artículo que lo dio a conocer (ibídem), ningún relieve, ni tampoco medidas. Esta tableta de arcilla presenta cuatro caras antropomorfas opuestas de dos en dos. Los que se hallan opuestos en el sentido longitudinal, se fusionan en el centro del rectángulo formando un motivo idéntico al motivo con bordes cóncavos clásico en las grandes urnas pintadas de Muitzentza. Las cuatro caras tienen ojos en rombos y también figuras en forma de flecha. Estos casos son también dos rasgos comunes en Muitzentza. Santiago Rivas Panduro y los otros autores del artículo sostienen que el objeto proviene de grupos zaparoanos, conocidos en la antigua Reducción de Pinches. Según los archivos jesuitas, esta zona estaba poblada de pinches, un grupo hoy en día extinto y reemplazado por los achuar y kichwa (Rivas Panduro et al., 2008: 282)

\section{CONCLUSIÓN}

El material de Muitzentza, todavía muy poco conocido, se caracteriza entre otras cosas, por la presencia de ollas pintadas sobre fondo natural o fondo engobado rojo. Estos ceramios fueron utilizados como urnas funerarias (entierro secundario probablemente) pero también en la vida doméstica, por ejemplo para almacenar la cerveza de mandioca. Una fecha obtenida de un basural precolombino indica una edad entre los siglos XI y XII d. C. La tradición cerámica tiene una dispersión aguas abajo de la alta cuenca del río Pastaza, es decir en la zona en donde el Pastaza se vuelve navegable. Por esta razón, proponemos la hipótesis de que las antiguas poblaciones portadoras de esta tradición cerámica jugaron un papel de intermediario entre la Amazonía de los caminos y senderos, y la Amazonía de los grandes ríos, drenada por el Marañón. Parece probable que esos pueblos pertenecieran al conjunto zaparoano (como los gaes y los pinches), que ocupaba la región al inicio de la Colonia. En todo caso, los intercambios 
hacia el sur con tradiciones relevantes del Horizonte corrugado son evidentes aunque permanecieran probablemente limitados. Una influencia del Horizonte polícromo, presente en los ríos Napo, Marañón y Ucayali, parece ser posible.

Por último, se hace obvio que esta nueva tradición cerámica abre nuevas perspectivas sobre el origen de la tradición cerámica de los actuales kichwa canelos de la provincia de Pastaza en el Oriente ecuatoriano, grupo que se formó durante la decadencia de los grupos zaparoanos.

\section{Agradecimientos}

Agradecemos a Warren DeBoer y Francisco Valdez por sus observaciones, críticas y correcciones. El texto ha sido también corregido por Belém Muriel.

\section{Referencias citadas}

BOLIAN, C. E., 1976 - Archaeological Excavations in the Trapecio of Amazonas: The Polychrome Tradition; Urbana-Champaign: Universidad de Illinois. Tesis de doctorado $(\mathrm{PhD})$.

DeBOER, W., 2003 - Ceramic Assemblage Variability in the Formative of Ecuador and Peru. In: Archaeology of Formative Ecuador (J. Scott Raymond \& R. L. Burger, eds.): 289336; Washington, D.C.: Dumbarton Oaks Research Library and Collection.

DeBOER, W., ROSS, E., ROSS, J. \& VEALE, M., 1977 - Two ceramic collections from Rio Huasaga, Northern Peru: their Place in the Prehistory of the Upper Amazon. El Dorado, 2 (2): 1-11.

DUCHE HIDALGO, C. \& SAUliEU, G. de, 2009 - Pastaza precolombino. Datos arqueológicos preliminares con el catálogo del Museo etno-arqueológico de Puyo y del Pastaza, 143 pp.; Quito: Abya Yala.

EVANS, C. \& MEGGERS, B., 1968 - Archaeological Investigations on the Rio Napo, Eastern Ecuador, xvi+127 pp.; Washington DC.: Smithsonian Institution Press.

FUNG PINEDA, R., 1981 - Notas y comentarios sobre el sitio de Valencia en el río Corrientes. Amazonía Peruana, IV-7: 99-137.

GUFFROY, J., 2006 - El Horizonte corrugado: correlaciones estilísticas y culturales. Bulletin de l'Institut français d'études andines, 35-3: 347-359.

HARNER, M., 1995 [1972] - Les Jivaros, Hommes des cascades sacrées, 253 pp.; París: Payot.

KARTSEN, R., 1935 - The Head Hunters of Western Amazonas. The life and Culture of the Jivaro Indians of Eastern Ecuador and Peru, 598 pp.; Helisingfors: Societas Scientiarum Fennica.

LATHRAP, D., 1970 - The upper Amazon, 256 pp.; New York: Praeger Publisher.

MARTINEAU, R., 2010 - Brunissage, polissage et degrés de séchage. Un référentiel expérimental. Les nouvelles de l'archéologie, 119, mars 2010: 13-19.

MORALES CHOCANO, D., 1991 - Chambira: alfareros tempranos en la Amazonía peruana. In: Estudios de la arqueología peruana (D. Bonnavia, ed.): 149-176; Lima: CONCYTEC. 
MYERS, T. \& DEAN, B., 1999 - Cerámica Prehispánica del río Chambira, Loreto. Amazonía Peruana, XIII-26: 255-288.

RAVINES, R., 1981 - Yacimientos arqueológicos en la région nororiental del Perú. Amazonía Peruana, IV-7: 99-137.

RAVINES, R., 1989 - Cerámica del río Morona, Alto Amazonas, Loreto. Boletín de Lima, 66: $14-18$.

REEVE, M. E., 2002 - Los Quichuas del Curaray. El proceso de formación de la identidad, 222 pp.; Quito: Abya Yala.

RIVAS PANDURO, S., MEDINA MENDOZA, A., ABANTO LLAQUE, J., RIOS ZUMAETA, R. \& CALDAS CARRILLO, C., 2008 - Arqueología de las cuencas del Pastaza y Morona. Reporte de zonificación ecológica económica. Amazonía Peruana, XV-31: 269-302.

ROSTAIN, S., 1999 - Occupations humaines et fonction domestique de monticules préhistoriques d'Amazonie équatorienne. Bulletin de la Société suisse des américanistes, 63: 71-95.

ROSTAIN, S., 2006 - Etnoarqueología de las casas Huapula y Jívaro. Bulletin de l'Institut français d'études andines, 35 (3): 1-10.

SAULIEU, G. de, 2006 - Revisión del material cerámico de la colección Pastaza (Amazonía ecuatoriana). Journal de la Société des américanistes, 92-1: 279-301.

TAYLOR, A. C., 1986 - Les versants orientaux des Andes septentrionales: des Bracamoros aux Quijos. In: L'Inca, I'Espagnol et les sauvages (F.-M. Renard-Casevitz, T. Saignes \& A. C. Taylors, eds.): 217-352; París: Association pour la Diffusion de la Pensée Française.

TAYLOR, A. C., 2010 - Voir comme un Autre : figurations amazoniennes de l'âme et du corps. In: La Fabrique des images. Visions du monde et formes de la représentation (P. Descola, ed.): 41-50; París: Musée du quai Branly, Somogy.

TRUJILLO, J., 2001 - Memorias del Curaray, 365 pp.; Quito: Fondo Populorum Progressio.

TESSMANN, G., 1999 [1930] - Los indígenas del Perú nororiental. Investigaciones fundamentales para un estudio sistemático de la cultura, 499 pp.; Quito: Abya Yala. 